\title{
The Chromatin Accessibility Complex: Chromatin Dynamics through Nucleosome Sliding
}

\author{
P.B. BECKER \\ Adolf-Butenandt-Institut, Molekularbiologie, Ludwig-Maximilians-Universität, \\ 80336 München, Germany
}

\begin{abstract}
Understanding chromatin structure and function in all its facets has been a fascinating challenge for generations of researchers. The organization of eukaryotic genomes into chromatin serves three functions. First and foremost, the packaging of DNA as chromatin involves neutralization of the negative charges in the phosphodiester backbone of DNA and its bending around molecular spools, the histone octamers, to form nucleosomes. Not only is this organization necessary for fitting a genome into the nuclear interior, but it also serves to protect the valuable genetic information from damage and instability. At the same time, chromatin organization hinders the interaction of DNA-binding regulators, thereby creating a globally repressed ground state of gene activity. The majority of all genes have to remain switched off in any given cell and primary mechanisms of tight repression build on a basic chromatin infrastructure. Finally, this organization creates opportunities for regulation: Utilization of the DNA - be it to transcribe genes, to replicate the genome, to recombine chromosomal segments, or to repair DNA damage - involves changing the occlusive chromatin environment, thereby allowing regulators to gain access to the substrate. Although nucleosomes are dynamic entities due to the very nature of the histone-DNA interactions (Widom 1999), the opening and closing of chromatin is not left to chance: A large number of enzymes dedicated to this task have been identified during recent years. Besides those that covalently modify histones to change their interaction properties (Jenuwein and Allis 2001), one interesting class of enzymes is fueled by chemical energy: They couple ATP hydrolysis to changing chromatin organization (Becker and Hörz 2002; Narlikar et al. 2002; Peterson 2002; Lusser and Kadonaga 2003). In broadest terms, the process of changing histone-DNA interactions in order to modulate the access of DNA is called "nucleosome remodeling." About 10 years ago we discovered a nucleosome remodeling entity, the Chromatin Accessibility Complex (CHRAC), during our search for an energy-dependent chromatin opening (Varga-Weisz et al. 1997). Since then, we and several other groups have studied the molecular anatomy of CHRAC and its mechanism of nucleosome remodeling. Although its physiological function has not been clearly established, all available results point to crucial functions of CHRAC for the assembly and maintenance of dynamic chromatin structure.
\end{abstract}

\section{IN SEARCH OF ATP-DEPENDENT NUCLEOSOME REMODELING ACTIVITIES}

Because of its potential for genetic analysis, Drosophila melanogaster is an excellent model organism suited for deciphering molecular mechanisms of eukaryotic gene function during development. Although less appreciated, Drosophila also provides a very good opportunity for biochemical studies. Drosophila embryos, which can be harvested in kilogram amounts from mass populations of fruit flies, are among the most active tissues. Extracts from embryos are rich in enzymatic activities, notably factors governing all aspects of gene expression. Relevant for the current discussion are the large quantities of chromatin assembly and nucleosome remodeling activities present in very early embryos. The first ten nuclear divisions during embryonic development occur very rapidly in the absence of zygotic transcription and protein synthesis. The assembly of the more than 1000 genomes arising into chromatin relies entirely on large stockpiles of histones, histone chaperones, and other assembly activities. Extracts from early embryos contain all these activities in proper stoichiometry and are, therefore, very competent in the assembly of experimental DNA into long nucleosomal arrays with physiological spacing. The assembly of such arrays also involves an ATP-dependent step. In the absence of ATP, nucleosomes will form, but their succession on DNA will be unordered and interrupted by gaps of free DNA (Becker and Wu 1992).

Chromatin reconstituted in this way contains many nonhistone proteins and is surprisingly transparent for DNA-binding proteins, despite its physiological nucleosome density. The discovery that the interaction of DNAbinding proteins with their target sequences in crude chromatin was much facilitated by the presence of hydrolyzable ATP (Pazin et al. 1994; Tsukiyama et al. 1994; Varga-Weisz et al. 1995; Wall et al. 1995) initiated searches for ATP-dependent nucleosome remodeling enzymes in diverse laboratories. The harvest was impressive. The Wu lab found the Nucleosome Remodeling Factor (NURF), a complex that can be targeted to promoters in chromatin to derepress transcription (Tsukiyama and Wu 1995; Xiao et al. 2001; Badenhorst et al. 2002). Focusing on chromatin assembly factors, Kadonaga and colleagues found ACF, an ATP-dependent remodeling factor that can improve chromatin assembly 
in vitro and in vivo (Ito et al. 1997; Fyodorov and Kadonaga 2002b; Fyodorov et al. 2004). Since we had hints about an untargeted nucleosome remodeling activity (see below), we devised an assay that made use of prokaryotic restriction enzymes as probes for access to DNA in chromatin and purified CHRAC from the system (VargaWeisz et al. 1995, 1997). Interestingly, all three protein complexes purified from fly embryo chromatin contained a common ATPase, ISWI, as the primary constituent of the nucleosome remodeling activity (Längst and Becker 2001b).

\section{NUCLEOSOME SLIDING CATALYZED BY CHRAC AND OTHER ISWI-CONTAINING COMPLEXES}

As the name suggests, the Chromatin Accessibility Complex generates access to DNA in chromatin allowing the interaction of DNA-binding proteins including the replication initiator T-antigen (Alexiadis et al. 1998) suggesting that chromatin might be disrupted or disassembled during the remodeling reaction. Surprisingly, however, it turned out that this is not the case. Rather, the ATP-dependent action of CHRAC improves the quality of chromatin, as judged from the regularity and extent of nucleosomal arrays (Varga-Weisz et al. 1997), consistent with a role for CHRAC as a chromatin assembly factor, rather than a disassembly factor. Chromatin assembly in the absence of ATP results in irregular successions of nucleosomes, which can be converted into arrays with regular spacing, a hallmark of physiological chromatin by CHRAC and ATP (Fig. 1). Interestingly, a long-range nucleosome spacing in vivo has been observed in conjunction with recruitment of CHRAC/ACF to the IL2R- $\alpha$ locus through interaction with the scaffolding protein SATB1 in thymocytes (Yasui et al. 2002).

The only way an increased accessibility of DNA in chromatin could be reconciled with this CHRAC activity was to assume that intact nucleosomes could be rendered mobile on DNA. This mobility would bring nucleosomal DNA into the more accessible linker between histone octamers for a fraction of time, exposing it to potential DNA-binding proteins.

We envision that nucleosome mobilization by CHRAC contributes to the assembly of chromatin fibers with regular nucleosome spacing, a requirement for folding of the nucleosomal chain into higher-order structures. Conceivably, its activity would not come to rest once this task has been achieved, but would continue to move nucleosomes, thereby endowing chromatin with fluidity and the flexibility required to respond to changing molecular environments (Becker 2002).

Evidence for the mobility of nucleosomes in Drosophila embryo chromatin had been obtained earlier. The type of chromatin reconstituted in this system depends on the experimental conditions. For example, increasing cation concentrations during assembly will lead to a gradual increase in internucleosomal distances, such that nucleosomal arrays with repeat lengths covering the entire physiological range from 160 to $>200$ base pairs

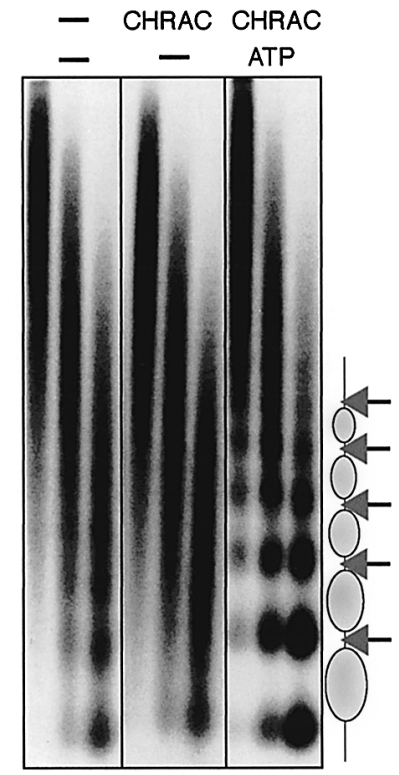

Figure 1. CHRAC catalyzes nucleosome spacing. Chromatin was assembled in an extract from Drosophila embryos in the absence of ATP. It was then treated with detergent in order to remove bound nucleosome remodeling activities. During further incubations, CHRAC and ATP were added as indicated. Treatment of the chromatin with Micrococcal Nuclease, which cleaves the internucleosomal linker DNA preferentially, reveals a regular "laddering" of fragments after gel electrophoresis only if the nucleosomes in the array are regularly spaced. A "smear" reveals the lack of ordering, irregular distances, and nucleosome-free gaps in chromatin. Details can be found in VargaWeisz et al. (1997).

(bp) are obtained (Blank and Becker 1995). Evidently, chromatin structures are flexible to accommodate various linker lengths during assembly. But how flexible are they afterward? In order to address this question we assembled arrays at high-salt conditions resulting in long linkers, followed by buffer change to lower ionic strength. Remarkably, we observed that the nucleosomal array changed in response to the altered conditions to accommodate a shorter repeat length (Fig. 2) (Varga-Weisz et al. 1995). The nucleosomal array was only flexible to respond to changes in the milieu if hydrolyzable ATP was present, pointing to an active, catalyzed process (Fig. 2). Since this assay monitored arrays of up to ten nucleosomes, all of which changed their positions relative to their neighbors, some nucleosomes must have moved over more than 100 base pairs. These and other experiments (Längst and Becker 2001b) with purified enzymes demonstrated that even extended arrays are still sufficiently flexible to allow nucleosomes to change their positions. Recently, Tsukiyama and colleagues provided the first evidence to suggest that ISWI-induced nucleosome sliding may also occur in vivo (Fazzio and Tsukiyama 2003). The discovery of ATP-dependent nucleosome mobility provided an elegant solution to the problem of how to ensure that regulatory proteins gain access to their binding sites while chromatin disruption is minimized (Becker 2002). 


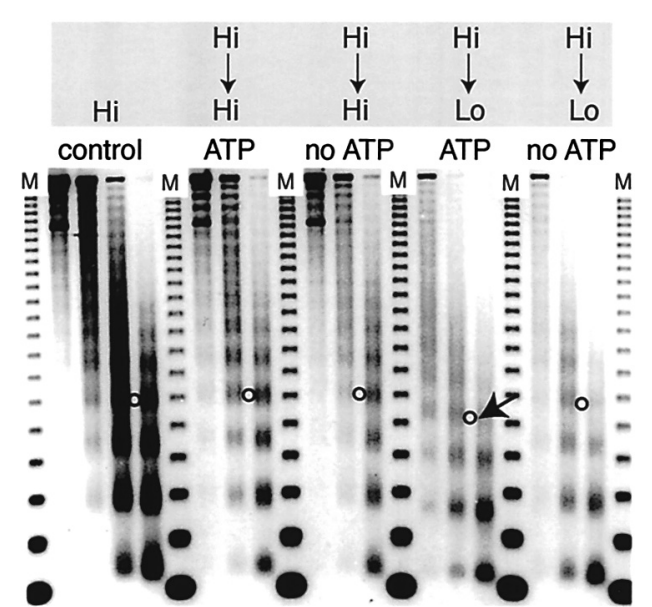

Figure 2. Nucleosomal arrays are dynamic. Chromatin was assembled in vitro at high-salt conditions, yielding long nucleosomal repeat lengths (NRLs) followed by buffer change to lower salt buffer. In the presence of ATP (and nucleosome remodeling factors) the positions of nucleosomes in the arrays relative to each other adjust to the altered ionic strength: All nucleosomes move closer together as seen by a shorter NRL. For details, see the text and Varga-Weisz et al. (1995). (Adapted, with permission, from Varga-Weisz et al. 1995 [@Nature Publishing Group] http://www.nature.com.)

The analysis of nucleosome mobility in more quantitative and mechanistic terms was facilitated by the development of an assay that allows observing the relocation of single histone octamers on DNA (Hamiche et al. 1999; Längst et al. 1999; Eberharter et al. 2004a). Wrapping of short DNA fragments around a single histone octamer frequently leads to different positions of the nucleosome with respect to the fragment ends. Particles that contain a more central nucleosome can be distinguished from those where the nucleosome is close to one fragment end by a simple electrophoretic mobility shift assay (Fig. 3). Because of the inherent stability of nucleosomes at low-salt conditions these different particles can be isolated and used as substrates for remodeling enzymes. CHRAC, ACF, NURF, and other ISWI-containing enzymes can catalyze movement of histone octamers on these short DNA fragments (Fig. 3) and the detailed characterization of this reaction demonstrated that histone octamers were moved as intact entities (nucleosome sliding) rather than being rapidly disand reassembled (Hamiche et al. 1999; Längst et al. 1999; Eberharter et al. 2001). Interestingly, although NURF can also slide nucleosomes, it is unable to function as a nucleosome spacing factor (Tsukiyama and Wu 1995).

\section{MOLECULAR ANATOMY OF CHRAC}

The enzyme responsible for the nucleosome remodeling activity of CHRAC is the ATPase ISWI, which also drives several other nucleosome remodeling complexes with distinct subunit composition (Längst and Becker 2001b; Corona and Tamkun 2004). In CHRAC, ISWI is associated with ACF1, a BAZ/WAL family member characterized by prominent PHD fingers and a bromodomain, and two histone-fold proteins of 14 and $16 \mathrm{kD}$
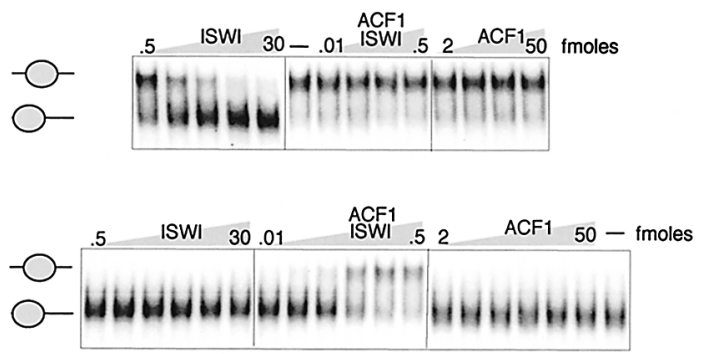

Figure 3. Interaction of ACF1 with ISWI modulates nucleosome mobilization. The nucleosome sliding assay monitors relocation of nucleosomes between central to peripheral positions on small DNA fragments. The cartoons to the left indicate which gel electrophoresis band corresponds to which type of particle. Association of ACF1 with ISWI changes the directionality of nucleosome sliding and at the same time increases the efficiency of the reaction: Far fewer femtomoles of remodeling complex are required to relocate nucleosomes in presence of ACF1 (for details see Eberharter et al. 2001). (Adapted, with permission, from Eberharter et al. 2001 [@Nature Publishing Group] http://www.nature.com.)

(CHRAC14 and CHRAC16) (Corona et al. 2000; Eberharter et al. 2001). Although topoisomerase II was initially thought to be an integral part of CHRAC (VargaWeisz et al. 1997), we later realized that this enzyme could be separated from CHRAC without affecting the activity or size of the complex (Eberharter et al. 2001). CHRAC is highly related to ACF, which solely consists of ISWI and ACF1 (Ito et al. 1999). CHRAC is a highly conserved entity: It has been isolated from human and frog cells (Poot et al. 2000; MacCallum et al. 2002) and recently it was found that the yeast ISW2 complex resembles CHRAC in its subunit composition (Iida and Araki 2004; McConnell et al. 2004). Metazoan CHRAC is organized by the large ACF1 subunit, which contacts ISWI at a central domain (Collins et al. 2002; Fyodorov and Kadonaga 2002a; Eberharter et al. 2004b) and binds the histone-fold proteins with an amino-terminal domain containing a sequence conservation termed the "WAC homology" (Kukimoto et al. 2004). The yeast protein Itc $1 p$, which is equivalent to ACF1 in the ISW2 complex, is dissimilar to ACF1 except for the WAC sequence in the amino terminus where the two histone-fold proteins interact (McConnell et al. 2004).

\section{MECHANISTIC CONSIDERATIONS}

The ATPase ISWI is a nucleosome remodeling factor by itself, which responds to the presence of the nucleosomal substrate with increased rates of ATP hydrolysis and induces the sliding of histone octamers on DNA (Corona et al. 1999; Längst et al. 1999; Clapier et al. 2001; Grüne et al. 2003). Currently, our favorite model for how ISWIcontaining nucleosome remodeling factors induce nucleosome sliding (Fig. 4) presumes that they function analogously to DNA translocases, such as helicases or restriction enzymes of type I, which move relative to DNA in a series of conformational changes triggered by nucleotide binding and/or hydrolysis (Längst and Becker 2004). If the enzyme was attached to a histone octamer, it could use analogous conformational choreography to 


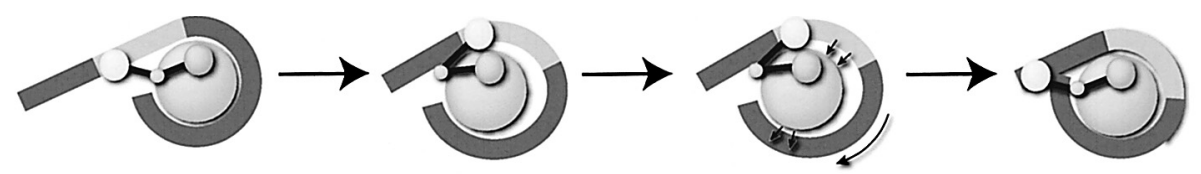

Figure 4. Hypothetical model of how nucleosome remodeling factors may mobilize histone octamers. The enzyme is depicted as two globular domains connected by a flexible hinge. The remodeler interacts with the histone body (large sphere) and the linker DNA. A conformational change in the enzyme pushes a segment of linker DNA (lighter gray) over the surface of the histone octamer.

"bulge up" the adjacent linker DNA and to "push" it into the realm of the nucleosome particle. Propagation of this loop over the histone surface, either by diffusion or through an active process (Haushalter and Kadonaga 2003), until it emerges at the opposite side of the particle, would effectively move the nucleosome relative to the DNA sequence (Fig. 4). While this model is consistent with many experimental observations (Havas et al. 2000; Längst and Becker 2001a, 2004; Becker and Hörz 2002), formal proof will require knowledge of the atomic structure of the remodeling machinery in contact with the nucleosome substrate as well as the characterization of reaction intermediates. Alternative potential outcomes of nucleosome remodeling reactions of the kinds observed for SWI/SNF-type remodelers, such as the disruption of histone-DNA interactions in the absence of nucleosome relocation (Fan et al. 2003), are less frequently observed with ISWI-containing enzymes.

The structure of a prominent substrate recognition domain within the carboxy-terminal half of ISWI, consisting of a DNA-binding SLIDE domain and a SANT domain, has recently been determined (Grüne et al. 2003). The SLIDE domain resembles classical helix-loop-helix DNA-binding domains in general and more specifically the DNA-binding modules of c-myb. ISWI preferentially interacts with the linker DNA adjacent to a nucleosome particle, a strategic position to initiate nucleosome remodeling since the DNA at its entry into the nucleosome is only loosely attached to the histone surface. SANT modules have been suggested to function as histone-tailbinding units in the context of other chromatin regulators (Boyer et al. 2004). However, even though ISWI requires the histone $\mathrm{H} 4$ tail for efficient nucleosome remodeling (Clapier et al. 2001, 2002; Hamiche et al., 2001), a strong, direct interaction of the enzyme with a histone tail has not yet been described. Interaction with the nucleosome substrate is likely to be complex since DNA and histone interactions of ACF1 have also been observed (Fyodorov and Kadonaga 2002a; Eberharter et al. 2004b) and the CHRAC14-CHRAC16 histone-fold dimer can also bind to DNA, albeit with modest affinity (Poot et al. 2000; Kukimoto et al. 2004; F. Hartlepp et al., in prep.). Furthermore, because of the nature of the remodeling process, these interactions are supposedly dynamic and presumably partially dependent on nucleotide-induced conformational changes of the enzyme.

\section{REGULATION OF ISWI ACTIVITY BY OTHER CHRAC SUBUNITS}

Although recombinant ISWI can carry out basic nucleosome sliding reactions, the enzyme is not very ef- ficient. Association of other CHRAC subunits profoundly improves the effectiveness of the reaction (i.e., the extent of remodeling per constant amount of ATP hydrolyzed). Interaction of ISWI with ACF1 improves nucleosome sliding efficiency by an order of magnitude (Fig. 3) (Ito et al. 1999; Eberharter et al. 2001). We recently found that this improved efficiency correlated with interactions of the ACF1 PHD fingers with the histone moiety within nucleosomes (Eberharter et al. 2004b). Conceivably, ISWI is a relatively inefficient remodeling enzyme because it lacks a stable anchoring point on the histone body. ACF1-histone contacts may provide such an anchor for the enzyme, assuring that the presumed conformational changes triggered by nucleotide binding and hydrolysis are efficiently converted into positional shifts of DNA relative to histones. Interestingly, association of ACF1 with ISWI not only boosts the efficiency of the enzyme, but it also changes the outcome of the remodeling reaction qualitatively (see Fig. 3). While ISWI alone moves nucleosomes from the center of a fragment to more peripheral positions (and not back), ACF (i.e., ISWI+ACF1) and CHRAC direct the converse relocation of end-positioned nucleosomes to more central positions (Fig. 3). While the significance of these differences in highly synthetic in vitro reactions remains to be established, it is nevertheless clear that the approach of ISWI to the nucleosome differs whether or not ACF1 is present.

The two small subunits CHRAC14 and CHRAC16 that differentiate CHRAC from ACF are of considerable interest, since their predicted (Corona et al. 2000) and experimentally determined structures (F. Hartlepp et al., in prep.) resemble that of a histone $\mathrm{H} 2 \mathrm{~A}-\mathrm{H} 2 \mathrm{~B}$ dimer pair. CHRAC14-CHRAC16 or their human counterparts, CHRAC17-CHRAC15, bind DNA with modest affinity (Poot et al. 2000; Kukimoto et al. 2004; F. Hartlepp et al., in prep.). Their dynamic interactions with structured DNA suggest that they may function as DNA chaperones in analogy to HMGB1 (see below). In vitro, the small subunits improve the activity of human and Drosophila ACF under conditions of limiting enzyme.

\section{REGULATION OF CHRAC ACTIVITY - TIP OF THE ICEBERG}

Very little is known about how CHRAC (or other ISWI-containing nucleosome remodeling factors) are regulated, but we assume that evolution has not missed the opportunity to control the level of chromatin dynamics. Among the many possibilities are posttranslational modifications of the remodeling machinery, which are being worked out at the moment. One other important parameter of chromatin dynamics is obviously the state of 
the substrate, chromatin, itself. The packaging of a nucleosomal fiber is mainly determined by interacting proteins: the linker histone $\mathrm{H} 1$, abundant nonhistone proteins, such as the HMG proteins, or more specialized chromatin regulators, such as the polycomb group proteins. The interaction of histone H1 with the DNA at the entry/exit of the nucleosome is known to impede the action of nucleosome remodeling machines of the SWI2/SNF2 type (Hill and Imbalzano 2000; Horn et al. 2002). However, early experiments with crude, reconstituted chromatin suggested that nucleosomal arrays retain their dynamic properties in the presence of physiological H1 levels (Varga-Weisz et al. 1995). The effect of linker histones on ISWI-dependent nucleosome remodeling has not been determined so far. Even if it turns out to be inhibitory, there are obvious ways of how it may be modulated. The interaction of $\mathrm{H} 1$ with nucleosomes may be weakened by phosphorylation of the linker histone (Horn et al. 2002), or H1 may be competed off the nucleosome by HMGB1 (Varga-Weisz et al. 1994). HMGB1 interacts with the nucleosomal linker much more dynamically than H1. Under conditions of limiting remodeling activity the association of HMGB1 with mononucleosomal substrates activates ACF- and CHRAC-dependent nucleosome mobilization (Bonaldi et al., 2002). This stimulatory effect of HMGB1 may be due to the "DNA chaperone" function of HMGB1, which could conceivably help to flexibilize the DNA at its entry into the nucleosome such that it can be more readily distorted and bound by the remodeling factor (Bonaldi et al. 2002; Travers 2003). We thus predict that substitution of HMGB1 for H1 should result in dramatic stimulation of nucleosome mobility.

Very little is known about the effect of histone modifications on nucleosome remodeling activity. ACF1 contains a bromodomain, which might serve to contact acetylated lysine residues and hence to discriminate between histones of different acetylation status within the nucleosome (Strahl and Allis 2000). But even the recombinant ATPase ISWI itself is able to sense certain modifications. For unknown reasons, efficient completion of the ISWI ATPase cycles requires a basic, DNA-bound patch on the amino terminus of histone H4. On peptide substrates, lysine acetylation close to this "ISWI response determinant" dampens the stimulation of its ATPase activity (Clapier et al., 2001, 2002; Hamiche et al. 2001).

\section{NUCLEOSOME REMODELING MACHINE IN SEARCH OF A FUNCTION}

Although mutation of the ATPase ISWI itself leads to a rich phenomenology of chromosome aberrations and changes in gene expression (Corona and Tamkun 2004), the fact that ISWI resides in at least one other complex (NURF) besides CHRAC/ACF precludes a straightforward correlation of effects and complexes (Längst and Becker 2001b; Tsukiyama 2002; Corona and Tamkun 2004). Monitoring the ACF1 subunit does not allow discrimination between functions of CHRAC or ACF, but nevertheless yields important information on a combined activity profile. Kadonaga and colleagues recently described their analysis of a genetic deficiency of ACF1 in flies (Fyodorov et al. 2004). A large fraction of the animals harboring a homozygous null mutation of the ACF1 gene die during the larval-pupal transition, but those that do survive show chromatin assembly defects and corresponding alleviation of chromatin-based repression mechanisms, such as polycomb-mediated silencing and position effect variegation (Fyodorov et al. 2004). The data are most consistent with a global role of CHRAC/ACF for the assembly of chromatin and chromosomes, which is expected to indirectly affect most nuclear functions. However, the flies lacking ACF1 also show phenotypes reminiscent of defects in trithorax group genes, which are involved in the regulation of homeotic genes during development, and thus more specific roles of the remodeling complex in gene regulation are still possible (Fyodorov et al. 2004).

The acceleration of $\mathrm{S}$ phase in embryonic and larval cells, which may be explained by a lesser hindrance of DNA synthesis in a defective and hence less repressive chromatin environment, could be an example for such an indirect effect (Fyodorov et al. 2004). However, direct roles for CHRAC/ACF in replication have been invoked from the analysis of other systems as well. In unresolved contrast to the acceleration of S phase in ACF1-deficient flies, Varga-Weisz and colleagues (Collins et al. 2002) found that ablation of ACF1 by RNA interference or expression of a dominant negative ACF in human cells resulted in a delay of cell-cycle progression, which was explained by a slowed replication of pericentric heterochromatin. This scenario would be more consistent with a role for CHRAC/ACF as chromatin accessibility factors, which facilitate the progress of the enzyme through a resistant chromatin environment. The effects were not seen if the cells were treated with 5-aza-deoxycytidine, a drug that, among other effects, inhibits the maintenance DNA methyltransferase (Collins et al. 2002). Since fly chromatin does not carry DNA methylation marks outside of a very narrow window in early embryonic development, the tasks of fly and human CHRAC/ACF may differ according to their chromatin environment.

Proper functional discrimination of CHRAC and ACF relies entirely on monitoring the role of the two small histone-fold subunits. The antibodies currently available against the metazoan subunits do not allow unambiguous localization of these factors in nuclei. However, the genetic analysis of the histone-like CHRAC subunits in yeast, Dls1p and Dpb4p, revealed that they are required for proper functioning of the ISW2 complex, which involves site-specific nucleosome positioning and repression of selected genes in synergisms with histone deacetylases (McConnell et al. 2004). In an interesting analogy, targeting of ISWI and ACF1 to the IL2R- $\alpha$ gene locus correlates with extensive ordering of chromatin and repression of the gene in mouse thymocytes (Yasui et al. 2002).

Since Isw2p is targeted to sites of action in the absence of the small subunits, these must play a role in the remodeling process itself, rather than in recruiting the re- 
modeling complex. Interestingly, Dpb4p is also a subunit of a DNA polymerase $\varepsilon$ complex, which stabilizes repressive chromatin environment at telomers, whereas the ISW2 complex (yCHRAC) maintains open chromatin at telomers (Iida and Araki 2004). The human DNA polymerase $\varepsilon$ has been colocalized to late-replicating heterochromatin, like hACF1 (Collins et al. 2002; Fuss and Linn 2002). Whether there is a functional interaction between the two complexes in heterochromatin and, if so, whether this is mediated by their shared subunit Dpb4p remain to be seen.

Several rather fundamental questions concerning CHRAC function remain to be answered. Do the two histone-fold subunits that distinguish CHRAC from ACF provide the remodeling complex with a different quality? Does CHRAC have global, untargeted roles in chromatin assembly and the maintenance of dynamic chromatin structure or is its physiological action limited locally by recruitment of the complex to sites of action such as replication forks or selected promoters? We have evidence from different model systems to support either scenario: The defect of ACF1 in the mutant fly points to a rather global function (Fyodorov et al. 2004), whereas experiments in yeast and mammalian cells suggest targeting principles (Yasui et al. 2002; McConnell et al. 2004). How does nucleosome mobilization work in an environment, where most nucleosomes are part of highly folded nucleosomal fibers? Does CHRAC/ACF affect higherorder chromatin structures, or is its action limited to mobilization of individual nucleosomes in a decondensed nucleosomal fiber? And if so, what are the activities and principles that allow productive interaction of the nucleosome remodeling machinery itself with its substrate? The rich phenomenology that has emerged from the analysis of nucleosome remodeling by CHRAC and ACF promises exciting answers to all these questions.

\section{ACKNOWLEDGMENTS}

I thank my colleagues who during recent years spent long hours studying CHRAC and chromatin dynamics in my laboratory: Thiemo Blank, Patrick Varga-Weisz, Davide Corona, Simona Ferrari, Karl Nightingale, Cedric Clapier, Edgar Bonte, Gernot Längst, Jan Brzeski, Anton Eberharter, Felix Hartlepp, and Roger Ferreira. I am also grateful for trust- and fruitful collaborations with John Tamkun, Marco Bianchi, Tiziana Bonaldi, Christoph Müller, Tim Grüne, John Widom, and Rein Aasland. I thank P. Varga-Weisz, G. Längst, and A. Eberharter for comments on the manuscript; P. Varga-Weisz and G. Längst for Figures 1 and 4, respectively. Work on CHRAC was supported by Deutsche Forschungsgemeinschaft through grants Be 1140/2-4, SFB 190, and SFB 594; the European Union through Network Grant HPRNCT-2000-00078; and Fonds of the Chemische Industrie.

\section{REFERENCES}

Alexiadis V., Varga-Weisz P.D., Bonte E., Becker P.B., and Gruss C. 1998. In vitro chromatin remodelling by chromatin accessibility complex (CHRAC) at the SV40 origin of DNA replication. EMBO J. 17: 3428 .

Badenhorst P., Voas M., Rebay I., and Wu C. 2002. Biological functions of the ISWI chromatin remodeling complex NURF. Genes Dev. 16: 3186.

Becker P.B. 2002. Nucleosome sliding: Facts and fiction. EMBO J. 21: 4749.

Becker P.B. and Hörz W. 2002. ATP-dependent nucleosome remodeling. Annu. Rev. Biochem. 71: 247.

Becker P.B. and Wu C. 1992. Cell-free system for assembly of transcriptionally repressed chromatin from Drosophila embryos. Mol. Cell. Biol. 12: 2241.

Blank T.A. and Becker P.B. 1995. Electrostatic mechanism of nucleosome spacing. J. Mol. Biol. 252: 305-313.

Bonaldi T., Längst G., Strohner R., Becker P.B., and Bianchi M.E. 2002. The DNA chaperone HMGB1 facilitates ACF/CHRACdependent nucleosome sliding. EMBO J. 21: 6865 .

Boyer L.A., Latek R.R., and Peterson C.L. 2004. The SANT domain: A unique histone-tail-binding module? Nat. Rev. Mol. Cell Biol. 5: 158 .

Clapier C.R., Nightingale K.P., and Becker P.B. 2002. A critical epitope for substrate recognition by the nucleosome remodeling ATPase ISWI. Nucleic Acids Res. 30: 649.

Clapier C.R., Längst G., Corona D.F., Becker P.B., and Nightingale K.P. 2001. Critical role for the histone H4 N terminus in nucleosome remodeling by ISWI. Mol. Cell. Biol. 21: 875.

Collins N., Poot R.A., Kukimoto I., Garcia-Jimenez C., Dellaire G., and Varga-Weisz P.D. 2002. An ACF1-ISWI chromatin-remodeling complex is required for DNA replication through heterochromatin. Nat. Genet .32: 627.

Corona D.F. and Tamkun J.W. 2004. Multiple roles for ISWI in transcription, chromosome organization and DNA replication. Biochim. Biophys. Acta 1677: 113.

Corona D.F., Längst G., Clapier C.R., Bonte E.J., Ferrari S., Tamkun J.W., and Becker P.B. 1999. ISWI is an ATP-dependent nucleosome remodeling factor. Mol. Cell 3: 239.

Corona D.F., Eberharter A., Budde A., Deuring R., Ferrari S., Varga-Weisz P., Wilm M., Tamkun J., and Becker P.B. 2000. Two histone fold proteins, CHRAC-14 and CHRAC-16, are developmentally regulated subunits of chromatin accessibility complex (CHRAC). EMBO J. 19: 3049.

Eberharter A., Langst G., and Becker P.B. 2004a. A nucleosome sliding assay for chromatin remodeling factors. Methods Enzymol. 377: 344.

Eberharter A., Vetter I., Ferreira R., and Becker P.B. 2004b. ACFI improves the effectiveness of nucleosome mobilization by ISWI through PHD-histone contacts. EMBO J. 23: 4029.

Eberharter A., Ferrari S., Längst G., Straub T., Imhof A., VargaWeisz P., Wilm M., and Becker P.B. 2001. Acf1, the largest subunit of CHRAC, regulates ISWI-induced nucleosome remodelling. EMBO J. 20: 3781 .

Fan H.Y., He X., Kingston R.E., and Narlikar G.J. 2003. Distinct strategies to make nucleosomal DNA accessible. Mol. Cell 11: 1311.

Fazzio T.G. and Tsukiyama T. 2003. Chromatin remodeling in vivo: Evidence for a nucleosome sliding mechanism. Mol. Cell 12: 1333 .

Fuss J. and Linn S. 2002. Human DNA polymerase epsilon colocalizes with proliferating cell nuclear antigen and DNA replication late, but not early, in S phase. J. Biol. Chem. 277: 8658.

Fyodorov D.V. and Kadonaga J.T. 2002a. Binding of Acf1 to DNA involves a WAC motif and is important for ACF-mediated chromatin assembly. Mol. Cell. Biol. 22: 6344.

- 2002b. Dynamics of ATP-dependent chromatin assembly by ACF. Nature 418: 897 .

Fyodorov D.V., Blower M.D., Karpen G.H., and Kadonaga J.T. 2004. Acfl confers unique activities to ACF/CHRAC and promotes the formation rather than disruption of chromatin in vivo. Genes Dev. 18: 170.

Grüne T., Brzeski J., Eberharter A., Clapier C.R., Corona D.F., Becker P.B., and Müller C.W. 2003. Crystal structure and functional analysis of a nucleosome recognition module of the remodeling factor ISWI. Mol. Cell 12: 449. 
Hamiche A., Sandaltzopoulos R., Gdula D.A., and Wu C. 1999. ATP-dependent histone octamer sliding mediated by the chromatin remodeling complex NURF. Cell 97: 833.

Hamiche A., Kang J.G., Dennis C., Xiao H., and Wu C. 2001 Histone tails modulate nucleosome mobility and regulate ATP-dependent nucleosome sliding by NURF. Proc. Natl. Acad. Sci. 98: 14316.

Haushalter K.A. and Kadonaga J.T. 2003. Chromatin assembly by DNA-translocating motors. Nat. Rev. Mol. Cell Biol. 4: 613.

Havas K., Flaus A., Phelan M., Kingston R., Wade P.A., Lilley D.M., and Owen-Hughes T. 2000. Generation of superhelical torsion by ATP-dependent chromatin remodeling activities. Cell 103: 1133.

Hill D.A. and Imbalzano A.N. 2000. Human SWI/SNF nucleosome remodeling activity is partially inhibited by linker histone H1. Biochemistry 39: 11649.

Horn P.J., Carruthers L.M., Logie C., Hill D.A., Solomon M.J., Wade P.A., Imbalzano A.N., Hansen J.C., and Peterson C.L. 2002. Phosphorylation of linker histones regulates ATP-dependent chromatin remodeling enzymes. Nat. Struct. Biol. 9: 263.

Iida T. and Araki H. 2004. Noncompetitive counteractions of DNA polymerase epsilon and ISW2/yCHRAC for epigenetic inheritance of telomere position effect in Saccharomyces cerevisiae. Mol. Cell. Biol. 24: 217.

Ito T., Bulger M., Pazin M.J., Kobayashi R., and Kadonaga J.T. 1997. ACF, an ISWI-containing and ATP-utilizing chromatin assembly and remodeling factor. Cell 90: 145.

Ito T., Levenstein M.E., Fyodorov D.V., Kutach A.K., Kobayashi R., and Kadonaga J.T. 1999. ACF consists of two subunits, Acf1 and ISWI, that function cooperatively in the ATP-dependent catalysis of chromatin assembly. Genes Dev. 13: 1529 .

Jenuwein T. and Allis C.D. 2001. Translating the histone code. Science 293: 1074.

Kukimoto I., Elderkin S., Grimaldi M., Oelgeschlager T., and Varga-Weisz P.D. 2004. The histone-fold protein complex CHRAC-15/17 enhances nucleosome sliding and assembly mediated by ACF. Mol. Cell 13: 265 .

Längst G. and Becker P.B. 2001a. ISWI induces nucleosome sliding on nicked DNA. Mol. Cell 8: 1085.

. 2001b. Nucleosome mobilization and positioning by ISWI-containing chromatin remodeling factors. J. Cell Sci. 114: 2561

- 2004. Nucleosome remodeling: one mechanism, many phenomena? Biochim. Biophys. Acta 1677: 58

Längst G., Bonte E.J., Corona D.F.V., and Becker P.B. 1999. Nucleosome movement by CHRAC and ISWI without disruption or trans-displacement of the histone octamer. Cell 97: 843.

Lusser A. and Kadonaga J.T. 2003. Chromatin remodeling by ATP-dependent molecular machines. Bioessays 25: 1192.

MacCallum D.E., Losada A., Kobayashi R., and Hirano T. 2002. ISWI remodeling complexes in Xenopus egg extracts: Identification as major chromosomal components that are regulated by INCENP-aurora B. Mol. Biol. Cell 13: 25.

McConnell A.D., Gelbart M.E., and Tsukiyama T. 2004. Histone fold protein Dls1p is required for Isw2-dependent chromatin remodeling in vivo. Mol. Cell. Biol. 24: 2605.

Narlikar G.J., Fan H.Y., and Kingston R.E. 2002. Cooperation between complexes that regulate chromatin structure and transcription. Cell 108: 475.

Pazin M.J., Kamakaka R., and Kadonaga J.T. 1994. ATP-dependent nucleosome reconfiguration and transcriptional activation from preassembled chromatin templates. Science 266: 2007.

Peterson C.L. 2002. Chromatin remodeling enzymes: Taming the machines. Third in review series on chromatin dynamics. EMBO Rep. 3: 319.

Poot R.A., Dellaire G., Hulsmann B.B., Grimaldi M.A., Corona D.F., Becker P.B., Bickmore W.A., and Varga-Weisz P.D. 2000. HuCHRAC, a human ISWI chromatin remodelling complex contains hACF1 and two novel histone-fold proteins. EMBO J. 19: 3377.

Strahl B.D. and Allis C.D. 2000. The language of covalent histone modifications. Nature 403: 41.

Travers A.A. 2003. Priming the nucleosome: A role for HMGB proteins? EMBO Rep. 4: 131.

Tsukiyama T. 2002. The in vivo functions of ATP-dependent chromatin-remodelling factors. Nat. Rev. Mol. Cell Biol. 3: 422.

Tsukiyama T. and Wu C. 1995. Purification and properties of an ATP-dependent nucleosome remodeling factor. Cell 83: 1011.

Tsukiyama T., Becker P.B., and Wu C. 1994. ATP-dependent nucleosome disruption at a heat-shock promoter mediated by binding of GAGA transcription factor. Nature 367: 525.

Varga-Weisz P.D., Blank T.A., and Becker P.B. 1995. Energydependent chromatin accessibility and nucleosome mobility in a cell-free system. EMBO J. 14: 2209.

Varga-Weisz P.D., van Holde K., and Zlatanova J. 1994. Competition between linker histones and HMG1 for binding to four-way junction DNA: Implications for transcription. Biochem. Biophys. Res. Commun. 203: 1904.

Varga-Weisz P.D., Wilm M., Bonte E., Dumas K., Mann M., and Becker P.B. 1997. Chromatin-remodelling factor CHRAC contains the ATPases ISWI and topoisomerase II (erratum in Nature [1997] 389: 1003). Nature 388: 598.

Wall G., Varga-Weisz P.D., Sandaltzopoulos R., and Becker P.B. 1995. Chromatin remodeling by GAGA factor and heat shock factor at the hypersensitive Drosophila hsp 26 promoter in vitro. EMBO J. 14: 1727.

Widom J. 1999. Equilibrium and dynamic nucleosome stability. Methods Mol. Biol. 119: 61.

Xiao H., Sandaltzopoulos R., Wang H., Hamiche A., Ranallo R., Lee K., Fu D., and Wu C. 2001. Dual functions of the largest NURF subunit NURF301 in nucleosome sliding and transcription factor interactions. Mol. Cell 8: 531.

Yasui D., Miyano M., Cai S., Varga-Weisz P., and KohwiShigematsu T. 2002. SATB1 targets chromatin remodelling to regulate genes over long distances. Nature 419: 641 . 


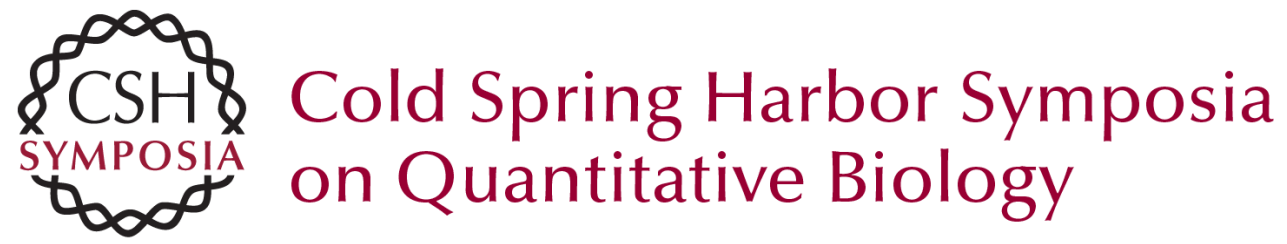

\title{
The Chromatin Accessibility Complex: Chromatin Dynamics through Nucleosome Sliding
}

\author{
P.B. BECKER
}

Cold Spring Harb Symp Quant Biol 2004 69: 281-288

Access the most recent version at doi:10.1101/sqb.2004.69.281

References This article cites 58 articles, 22 of which can be accessed free at: http://symposium.cshlp.org/content/69/281.full.html\#ref-list-1

\section{License}

Email Alerting

Receive free email alerts when new articles cite this article - sign up in Service the box at the top right corner of the article or click here. 